\title{
Composição nutricional e avaliação de rótulo de rações secas para cães comercializadas em Jaboticabal-SP
}

[Nutritional composition and label evaluation of dry dog foods sold in Jaboticabal-SP]

\author{
A.C. Carciofi ${ }^{1}$, R.S. Vasconcellos ${ }^{2}$, N.C. Borges ${ }^{2}$, J.V. Moro ${ }^{3}$, F. Prada ${ }^{4}$, V. O. Fraga ${ }^{5}$. \\ ${ }^{1}$ Faculdade de Ciências Agrárias e Veterinárias - UNESP \\ Via de Acesso Prof. Paulo Donato Castellane, s/n \\ 14884-900 - Jaboticabal, SP \\ ${ }^{2}$ Aluno de pós-graduação - FCAV - UNESP - Jaboticabal, SP \\ ${ }^{3}$ Aluna de pós-graduação - FCAV - UNESP - Jaboticabal, SP \\ ${ }^{4}$ Faculdade de Medicina Veterinária e Zootecnia - USP - São Paulo, SP \\ ${ }^{5}$ Residente - FCAV-UNESP - Jaboticabal, SP
}

\begin{abstract}
RESUMO
Avaliaram-se 49 marcas de rações para cães adultos e filhotes comercializadas em Jaboticabal-SP. Os alimentos foram divididos em três segmentos: econômico, standard e super-premium. Nessa ordem, as rações para cães adultos apresentaram, em média, 16,9\%, 20,9\% e 27,8\% de proteína, 9,7\%, 10,5\% e 15\% de gordura, $6,4 \%, 2,9 \%$ e $1,1 \%$ de fibra e 1,9\%, 1,9\% e 1,4\% de Ca. Para filhotes, os produtos standard e super-premium apresentaram, respectivamente, $26,1 \%$ e $31,0 \%$ de proteína, $10,8 \%$ e $15,2 \%$ de gordura, $2,6 \%$ e $2,4 \%$ de fibra, $2,1 \%$ e $1,7 \%$ de $\mathrm{Ca}$ e $1,6 \%$ e $1,3 \%$ de $\mathrm{P}$. A porcentagem de rações cujos teores nutricionais declarados no rótulo não estavam de acordo com os encontrados nas análises de laboratório foi, para os produtos super-premium para filhotes, $80,0 \%$ para o Ca e 60,0\% para a gordura; para os produtos standard para filhotes, $28,6 \%$ para proteína e 57,2\% para o cálcio; para os produtos econômicos para cães adultos, 44,0\% para a fibra e 33,0\% para a proteína; para os produtos standard para cães adultos de $33,0 \%$ para a gordura e 50,0\% para o Ca; e para os produtos super-premium para cães adultos, 50,0\% para o cálcio e 33,0\% para a gordura. Foram encontradas inadequações nutricionais em produtos, como teores insuficientes de proteína e altas concentrações de fibra, cálcio e fósforo.
\end{abstract}

Palavras-chave: cão, nutrição, ração, nutrientes, análise de rótulo, Brasil

\begin{abstract}
Fortynine food products for adult or juvenile dogs, commercially available in Jaboticabal, São Paulo, Brazil, were tested for nutrient composition. The products were divided into three categories: low-cost, standard and super-premium. In that order, average compositions for adult foods were 16.9\%, 20.9\% and $27.8 \%$ protein, $9.7 \%, 10.5 \%$ and $15 \%$ fat, $6.4 \%, 2.9 \%$ and $1.1 \%$ fiber, and $1.9 \%, 1.9 \%$ and $1.4 \%$ calcium. For puppy foods, the average compositions of standard and super-premium foods were $26.1 \%$ and $31 \%$ protein, $10.8 \%$ and $15.2 \%$ fat, $2.6 \%$ and $2.4 \%$ fiber, $2.1 \%$ and $1.7 \% \mathrm{Ca}$, and $1.6 \%$ and $1.3 \% \mathrm{P}$, respectively. The percentages of products whose published label values were in disagreement with laboratory results were: super-premium products for puppies, $80 \%$ for Ca and $60 \%$ for fat; standard products for puppies, 28.6\% for protein and 57.2\% for Ca; low-cost products for adults, 44\% for fiber and 33\% for protein; standard products for adults, 33\% for fat and 50\% for Ca; super-premium products for adults, 50\% for calcium and 33\% for fat. Products with nutritional shortcomings were found, such as insufficient protein content and too high levels of fiber, calcium, and phosphorus.
\end{abstract}

Keywords: dog, dog food, nutrient composition, label analysis, Brazil

Recebido em 17 de março de 2004

Aceito em 25 de novembro de 2005

E-mail: aulus@fcav.unesp.br 


\section{INTRODUÇÃO}

Segundo a Associação Nacional dos Fabricantes de Alimentos para Animais, existem no Brasil cerca de 21 milhões de cães com endereço fixo, a segunda maior população do planeta. Destes, $34 \%$ são alimentados com ração industrializada. A produção de alimentos para animais de estimação foi da ordem de 1.234.000 toneladas no ano de 2002, mantendo crescimento médio anual de $5 \%$ nos últimos 10 anos e com potencial produtivo estimado de 3.200.000 toneladas/ano (Prior, 2003). Existem atualmente no país mais de 300 marcas registradas, produzidas por mais de 70 fabricantes, com preço e qualidade variáveis.

No Brasil, a responsabilidade da regulamentação das rações para cães e gatos é do Ministério da Agricultura, Pecuária e Abastecimento, prevista no Decreto $\mathrm{n}^{\mathrm{o}} 76.986$ de 6 de janeiro de 1976, que regulamentou a Lei $\mathrm{n}^{\circ} 6.198$, de 26 de dezembro de 1974, que dispõe sobre a inspeção e a fiscalização dos produtos destinados à alimentação animal. O Decreto é instruído por meio de Instruções Normativas, que são atualizadas e publicadas periodicamente. A Instrução Normativa $\mathrm{n}^{\circ}$ 08, publicada em 2002 e em vigor até a data da elaboração deste trabalho, fixa e identifica as características mínimas de qualidade a que devem obedecer os alimentos completos e os especiais para cães e gatos.

Os alimentos industrializados são divididos de acordo com a segmentação comercial instituída pela própria indústria, não caracterizada ou contida na Instrução Normativa $\mathrm{n}^{\circ} 08$, e baseia-se na qualidade e no tipo de matéria-prima, concentração de nutrientes, características do rótulo e preço, sendo normalmente aceita pelos consumidores como um critério qualitativo que norteia decisões de compra (Carciofi, 2003).

Este trabalho teve por objetivos determinar a composição nutricional e avaliar as informações declaradas no rótulo de alimentos secos para cães adultos e filhotes, de diferentes segmentos comerciais.

\section{MATERIAL E MÉTODOS}

Foram adquiridas entre os meses de julho e novembro de 2002, aleatoriamente, 49 marcas de rações para cães, sendo 30 para adultos e 19 para filhotes, fabricadas por 18 empresas diferentes, comercializadas em casas de rações e pet shops da cidade de Jaboticabal-SP. Elas foram identificadas por números para se preservar a privacidade do fabricante. As rações para cães adultos foram classificadas em econômicas, standard e super-premium, baseando-se nos critérios de Case et al. (1998). Em relação às rações para filhotes, foram analisados apenas produtos standard e super-premium.

Determinaram-se os teores de proteína bruta (PB), extrato etéreo hidrólise ácida (EEA), matéria mineral (MM), matéria seca (MS), fibra bruta $(\mathrm{FB})$, cálcio $(\mathrm{Ca})$ e fósforo $(\mathrm{P})$. As rações foram moídas em micro-moinho em peneira de $1 \mathrm{~mm}$ e processadas em duplicata segundo metodologia compatível com a Association of the Official Analytical Chemists (Cunnif, 1997).

Os valores observados (VO) foram comparados com os valores declarados (VD) no rótulo pelo fabricante. Adotando-se uma tolerância de 10\% na análise, de acordo com a legislação em vigor, criou-se o seguinte critério de classificação quanto à adequação de rótulo: em conformidade (C)- rações que apresentaram resultados da análise laboratorial de acordo com os valores declarados no rótulo; em não conformidade (NC)- rações que apresentaram resultados da análise não de acordo com os valores declarados.

Os resultados das análises de laboratório foram, ainda, comparados com os valores mínimos e máximos permitidos pelo Ministério da Agricultura, Pecuária e Abastecimento (Brasil, 2002) e com as recomendações nutricionais da Association of American Feed Control Officials (Association... 2003), na matéria seca.

\section{RESULTADOS E DISCUSSÃO}

A composição nutricional das rações e os valores máximo e mínimo são apresentados na Tab. 1. A adequação dos rótulos foi tabulada, para cada nutriente, como a porcentagem de produtos em NC. 
Composição nutricional...

Tabela 1. Teores nutricionais declarados e observados, em porcentagem, e adequação de rótulo de alimentos secos para cães, de diferentes categorias comerciais, adquiridos em Jaboticabal - SP

\begin{tabular}{|c|c|c|c|c|c|}
\hline & \multicolumn{3}{|c|}{ Adultos } & \multicolumn{2}{|c|}{ Filhotes } \\
\hline & $\begin{array}{l}\text { Econômico } \\
(\mathrm{n}=9)\end{array}$ & $\begin{array}{c}\text { Standard } \\
(\mathrm{n}=15)\end{array}$ & $\begin{array}{c}\text { Super-premium } \\
(\mathrm{n}=6)\end{array}$ & $\begin{array}{l}\text { Standard } \\
(\mathrm{n}=14)\end{array}$ & $\begin{array}{c}\text { Super-premium } \\
(\mathrm{n}=5)\end{array}$ \\
\hline & \multicolumn{5}{|c|}{ Umidade (\%) } \\
\hline $\mathrm{VD} \pm \mathrm{DP}$ & $12,0 \pm 0,0$ & $12,0 \pm 0,0$ & $10,7 \pm 1,0$ & $12,0 \pm 0,0$ & $10,9 \pm 1,0$ \\
\hline $\mathrm{VO} \pm \mathrm{DP}$ & $8,2 \pm 1,1$ & $8,0 \pm 1,2$ & $6,0 \pm 0,6$ & $7,8 \pm 0,9$ & $6,9 \pm 1,2$ \\
\hline$(\min -\max )$ & $(5,8-9,8)$ & $(6,5-11,0)$ & $(6,0-7,4)$ & $(6,0-9,3)$ & $(5,0-8,3)$ \\
\hline \multirow[t]{2}{*}{$\mathrm{NC}(\%)$} & - & - & - & - & - \\
\hline & \multicolumn{5}{|c|}{ Proteína bruta $(\%)$} \\
\hline $\mathrm{VD} \pm \mathrm{DP}$ & $18,3 \pm 1,4$ & $20,9 \pm 1,5$ & $27,2 \pm 4,5$ & $26,9 \pm 1,5$ & $29,8 \pm 0,4$ \\
\hline $\mathrm{VO} \pm \mathrm{DP}$ & $16,9 \pm 2,4$ & $20,3 \pm 1,9$ & $27,8 \pm 4,5$ & $26,1 \pm 2,3$ & $31,0 \pm 3,9$ \\
\hline$(\min -\max )$ & $(12-19,6)$ & $(17,4-23,1)$ & $(22,7-36,0)$ & $(22,6-29,0)$ & $(26,4-36,8)$ \\
\hline \multirow[t]{2}{*}{$\mathrm{NC}(\%)$} & 33,3 & 14,3 & - & 28,6 & 20,0 \\
\hline & \multicolumn{5}{|c|}{ Extrato etéreo hidrólise acida (\%) } \\
\hline $\mathrm{VD} \pm \mathrm{DP}$ & $5,2 \pm 0,6$ & $6,5 \pm 1,2$ & $14,3 \pm 2,3$ & $8,6 \pm 2,0$ & $16,2 \pm 3,9$ \\
\hline $\mathrm{VO} \pm \mathrm{DP}$ & $9,7 \pm 1,2$ & $10,5 \pm 2,1$ & $15,0 \pm 2,5$ & $10,8 \pm 1,7$ & $15,2 \pm 3,0$ \\
\hline$(\min -\max )$ & $(8,0-11,8)$ & $(6,6-12,7)$ & $(10,8-17,1)$ & $(9,6-14,7)$ & $(11,7-19,2)$ \\
\hline \multirow[t]{2}{*}{$\mathrm{NC}(\%)$} & - & - & 33,6 & 7,1 & 60,0 \\
\hline & \multicolumn{5}{|c|}{ Fibra bruta (\%) } \\
\hline $\mathrm{VD} \pm \mathrm{DP}$ & $6,3 \pm 0,9$ & $5,3 \pm 1,1$ & $3,3 \pm 0,9$ & $4,0 \pm 0,7$ & $3,2 \pm 0,4$ \\
\hline $\mathrm{VO} \pm \mathrm{DP}$ & $6,4 \pm 3,7$ & $2,9 \pm 2,1$ & $1,1 \pm 0,1$ & $2,6 \pm 2,0$ & $2,4 \pm 2,3$ \\
\hline$(\min -\max )$ & $(1,8-12,4)$ & $(1,2-8,2)$ & $(0,9-1,3)$ & $(0,6-7,5)$ & $(0,6-6,4)$ \\
\hline \multirow[t]{2}{*}{$\mathrm{NC}(\%)$} & 44,4 & 7,1 & - & 14,2 & 20,0 \\
\hline & \multicolumn{5}{|c|}{ Matéria mineral (\%) } \\
\hline $\mathrm{VD} \pm \mathrm{DP}$ & $10,3 \pm 2,1$ & $9,5 \pm 2,2$ & $7,3 \pm 1,4$ & $9,8 \pm 1,5$ & $8,2 \pm 1,0$ \\
\hline $\mathrm{VO} \pm \mathrm{DP}$ & $9,0 \pm 3,3$ & $8,1 \pm 1,0$ & $6,3 \pm 0,8$ & $8,5 \pm 1,2$ & $7,6 \pm 1,1$ \\
\hline$(\min -\max )$. & $(6,7-17,1)$ & $(5,8-11,4)$ & $(5,1-7,3)$ & $(9,6-14,7)$ & $(7,1-9,0)$ \\
\hline \multirow[t]{2}{*}{$\mathrm{NC}(\%)$} & 11,1 & - & 16,7 & - & 20,0 \\
\hline & \multicolumn{5}{|c|}{ Cálcio (\%) } \\
\hline $\mathrm{VD} \pm \mathrm{DP}$ & $1,9 \pm 0,3$ & $1,9 \pm 0,5$ & $1,4 \pm 0,3$ & $1,9 \pm 0,3$ & $1,4 \pm 0,1$ \\
\hline $\mathrm{VO} \pm \mathrm{DP}$ & $1,9 \pm 0,6$ & $1,9 \pm 0,5$ & $1,4 \pm 0,3$ & $2,1 \pm 0,6$ & $1,7 \pm 0,3$ \\
\hline$(\min -\max )$ & $(1,3-3,3)$ & $(0,9-3,0)$ & $(0,9-1,7)$ & $(1,2-3,2)$ & $(1,3-2,1)$ \\
\hline \multirow[t]{2}{*}{$\mathrm{NC}(\%)$} & 11,1 & 50,0 & 50,0 & 57,2 & 80,0 \\
\hline & \multicolumn{5}{|c|}{ Fósforo (\%) } \\
\hline $\mathrm{VD} \pm \mathrm{DP}$ & $0,7 \pm 0,2$ & $0,9 \pm 0,2$ & $0,8 \pm 0,2$ & $1,0 \pm 0,1$ & $0,9 \pm 0,1$ \\
\hline $\mathrm{VO} \pm \mathrm{DP}$ & $1,5 \pm 0,5$ & $1,5 \pm 0,5$ & $1,0 \pm 0,2$ & $1,6 \pm 0,4$ & $1,3 \pm 0,5$ \\
\hline$(\min -\max )$ & $(0,8-2,2)$ & $(0,8-2,6)$ & $(0,8-1,2)$ & $(1,0-2,4)$ & $(1,0-2,2)$ \\
\hline
\end{tabular}

VD \pm DP: valor declarado médio \pm desvio-padrão; VO \pm DP: valor observado médio \pm desvio-padrão; NC: porcentagem de produtos com nutrientes em não conformidade.

A PB diferiu 4 a 7 pontos percentuais entre os segmentos comerciais de produtos. Os teores de EEA das rações para cães adultos dos segmentos econômico e standard foram próximos entre si. $\mathrm{O}$ segmento super-premium apresentou, aproximadamente, 5 pontos percentuais a mais de EEA que os demais. Os VO de EEA dos produtos econômicos foram 4,5 pontos percentuais acima dos VD. A concentração de EEA foi semelhante entre os produtos para filhotes e adultos, os produtos econômicos apresentaram 2,2 vezes mais FB que os standard, que, por sua vez, apresentaram 2,6 vezes mais FB que os super-premium. Os teores de Ca e P foram maiores nos produtos econômico e standard e menores nos super-premium, tanto nos produtos para animais adultos como nos para filhotes.
Os valores nutricionais médios encontrados atenderam, em geral, às exigências da Instrução Normativa $n^{\circ} 8$. Destacaram-se, apenas, o alto teor de FB médio dos produtos econômicos para cães adultos, perto do limite máximo permitido, e a alta concentração média de $\mathrm{Ca}$ dos produtos standard para cães filhotes, 2,1\%, acima do indicado pela Instrução Normativa $\mathrm{n}^{\circ} 8$.

Em relação às recomendações nutricionais mínimas e máximas preconizadas pela Association... (2003), constataram-se rações com problemas de formulação ou de controle e qualidade. Trinta e três por cento das rações para cães adultos do segmento econômico apresentaram PB inferior a $18 \%$ na MS, mínimo recomendado. Excesso de $\mathrm{Ca}$, ou seja, mais de $2,5 \%$ do elemento na MS, foi verificado em 
$11,1 \%$ dos produtos econômicos para cães adultos, em 26,7\% dos alimentos standard para cães adultos e em 42,9\% das rações standard para filhotes.

Em relação ao $\mathrm{P}, 55,6 \%$ das rações econômicas para cães adultos, $55,3 \%$ das standard para cães adultos, $64,3 \%$ dos produtos standard para filhotes e $20 \%$ dos alimentos super-premium para cães filhotes avaliados nesta amostragem apresentaram concentrações superiores a 1,6\% sobre a MS, máximo recomendado pela Association... (2003). Destaque-se que essa não é uma avaliação quanto à correspondência entre o valor declarado no rótulo e o valor constatado no produto e sim uma comparação entre os teores nutricionais efetivamente praticados os adequados nutricionalmente.

A ingestão excessiva de cálcio leva a competições desse macro-elemento com outros minerais, como o zinco, durante a absorção intestinal, o que pode ocasionar deficiência de zinco (Scott et al., 1996). A alta concentração de cálcio verificada em algumas rações pode ter participação no aumento da incidência de doenças osteoarticulares do desenvolvimento, como osteocondrose, osteocondrites, displasias de cotovelo e coxofemoral, constatadas na rotina clínica no Brasil por Pinto (2002). Schoenmakers et al. (1999) e Lauten et al. (2002), ao trabalharem respectivamente, com teores de cálcio de $3,1 \%$ e $2,7 \%$ na matéria seca, inferiores aos encontrados em alguns produtos na presente amostragem, reproduziram distúrbios osteoarticulares relacionados ao crescimento em cães de raças grandes. Hazewinkel et al. (1991) constataram que dietas com $3,3 \%$ de $\mathrm{Ca}$, independentemente de sua relação com o $\mathrm{P}$, promoveram maior taxa de retenção do $\mathrm{Ca} \mathrm{em}$ cães de raças grandes em fase de crescimento. As concentrações de $\mathrm{Ca}$ e $\mathrm{P}$ nas dietas de filhotes até seis meses de idade refletem-se rápida $\mathrm{e}$ diretamente sobre o seu conteúdo mineral ósseo, motivo pelo qual as doenças esqueléticas do desenvolvimento manifestam-se com maior freqüência no início do crescimento dos cães (Lauten et al., 2002).

Excesso de P, desbalanço nutricional de ocorrência mais freqüente na presente amostragem, pode ser particularmente deletério durante o crescimento e senilidade. Tryfonidou et al. (2003) demonstraram em filhotes de raças gigantes que a reabsorção renal de fósforo inorgânico é maior que a de filhotes de raças pequenas, explicando a retenção orgânica de $\mathrm{P}$ e a elevação da fosfatemia verificadas em animais alimentados com excesso de P. Acredita-se que ao redor de $25 \%$ dos cães idosos apresentem algum comprometimento da função renal (Crowell e Finco, 1975; Debraekeleer et al., 1999), de modo que a ingestão excessiva de $P$ deve ser evitada para animais nessa faixa etária. Redução da progressão da doença renal, aumento da qualidade e expectativa de vida e regularização das alterações bioquímicas relativas à insuficiência renal crônica foram conseguidas com dietas que apresentavam ao redor de 0,4\% de P (Kronfeld, 1994; Finco et al., 1992), valor correspondente a apenas $25 \%$ da concentração média encontrada nas rações avaliadas neste trabalho.

A relação Ca:P média (mínima; máxima) dos alimentos econômicos para cães adultos foi 1,3:1 $(0,7: 1 ; 2,5: 1)$, dos produtos standard para cães adultos, 1,3:1 (1:1; 1,9:1), dos super-premium para cães adultos, $1,3: 1 \quad(1,2: 1 ; 1,4: 1)$, dos produtos standard para filhotes, 1,3:1 (0,65:1; 1,8:1) e dos produtos super-premium para filhotes, 1,3:1 $(0,9: 1 ; 1,6: 1)$. Os valores médios estão adequados, mas alguns produtos apresentaram relação Ca:P adversa. Hazewinkel et al. (1991) e Schoenmakers et al. (2000), ao trabalharem com dietas para crescimento de cães que apresentavam concentrações e relações Ca:P adversas, mostraram alterações na ossificação endocondral, diminuição da taxa de crescimento, alterações na fosfatemia e elevação da creatinina, uréia e fosfatase alcalina séricas.

$\mathrm{O}$ excesso de minerais, especificamente $\mathrm{Ca}$ e $\mathrm{P}$, verificado neste trabalho pode ser atribuído às fontes de proteína empregadas na fabricação das rações. Um dos critérios para a avaliação dos ingredientes protéicos de origem animal é sua relação proteína:matéria mineral (Cowell et al., 2000). Quanto mais matéria mineral, representada pela maior participação de ossos na composição do ingrediente, normalmente menor sua digestibilidade. Além desse aspecto, farinhas protéicas com excesso de minerais apresentam limitações de inclusão na fórmula, pois carreiam consigo grande quantidade de $\mathrm{Ca}$ e $\mathrm{P}$. Como exemplo, citam-se dois ingredientes protéicos largamente empregados na fabricação de alimentos para cães: farinha de carne e ossos 
com $45 \%$ de $\mathrm{PB}, 11,3 \%$ de $\mathrm{Ca}$ e $5,9 \%$ de $\mathrm{P}$ e farinha de vísceras de frango com $58 \%$ de $\mathrm{PB}$, $4,4 \%$ de $\mathrm{Ca}$ e $2,6 \%$ de $\mathrm{P}$ (Rostagno et al., 2000). Para se acrescentar $10 \%$ de proteína à dieta por meio da farinha de carne e ossos, adiciona-se em torno de $2,5 \%$ de $\mathrm{Ca}$, ao passo que com farinha de vísceras de frango apenas $0,8 \%$.

Huber et al. (1986) demonstraram diminuição da digestibilidade da $\mathrm{PB}$, matéria seca e extrato etéreo em rações para cães com mais de $5 \%$ de FB. Concentrações acima de 5\% de FB foram encontradas em alimentos econômicos para cães adultos e standard para cães adultos e filhotes. $\mathrm{O}$ VO médio de FB dos produtos econômicos para cães adultos foi de $6,4 \%$, o que é agravado pelo fato de um terço dessas rações apresentarem proteína insuficiente. Esses autores verificaram, ainda, diferença significativa na taxa de crescimento, qualidade da pelagem e características hematológicas e bioquímicas dos cães alimentados com rações com alta fibra. Resultado semelhante foi encontrado por Burrows et al. (1982), que correlacionaram inversamente o conteúdo de celulose do alimento com sua digestibilidade e por Fahey et al. (1992), que demonstraram diminuição linear da digestibilidade da energia com a adição de fibra de aveia à ração. Soma-se a esse aspecto o fato de a fibra poder interferir negativamente sobre a absorção de macro e micro-elementos (Gross et al., 2000).

A elevada concentração de fibra, especialmente nos produtos de menor valor agregado, está afeita provavelmente ao tipo de ingrediente empregado. Nele existe maior participação de farelos vegetais ricos em fibra, como farelo de trigo (11\% de FB), farelo de arroz (11\% de FB) e farelo de soja $(6,5 \%$ de FB), utilizados para reduzir o custo do produto.

Embora não se tenha realizado ensaio para a determinação da energia metabolizável dos alimentos, devido à elevada concentração de FB verificada para alguns segmentos de mercado, acredita-se que as fórmulas recomendadas pelo Nutrient... (1985), Association... (2003) e Brasil (2002) possam estar superestimando a densidade energética de alimentos do segmento econômico e subestimando a dos super-premium. Kienzle et al. (1998) desenvolveram equações que parecem estimar mais corretamente o teor de EM das rações, pois consideram no cálculo o teor de FB do alimento uma alternativa interessante a ser explorada em virtude da grande variação na composição centesimal dos produtos demonstrada nesta amostragem.

Quanto ao cumprimento de garantia do rótulo, $44,4 \%$ dos produtos econômicos para cães adultos apresentaram mais $\mathrm{FB}$ e $33,3 \%$ menos $\mathrm{PB}$ do que declararam (Tab. 1). Considerando que os valores declarados já são limites, percebem-se, nesses casos, alimentos com qualidade comprometida, pois, além de apresentarem teor protéico insuficiente, existe uma relação inversa entre o teor de fibra e a digestibilidade da proteína da ração (Burrows et al., 1982; Kienzle et al., 2001). Em relação aos produtos standard para cães adultos, 14,3\% estavam em não conformidade quanto à proteína e metade deles com mais cálcio do que foi declarado pelo fabricante. Os produtos superpremium para cães adultos apresentaram não conformidade em $50 \%$ para o $\mathrm{Ca}$ e problemas quanto ao EEA, com 33,6\% apresentando valores inferiores aos declarados.

No segmento standard para filhotes, 14,2\% apresentaram mais FB, 28,6\% menos PB e $57,2 \%$ mais $\mathrm{Ca}$ que o declarado. Nos produtos super-premium, 20\% continham menos PB, 20\% mais FB, $60 \%$ não apresentavam a concentração de EEA declarada e $80 \%$ mais $\mathrm{Ca}$ do que o indicado. Deve-se considerar que a amostragem do segmento super-premium, com seis produtos avaliados para cães adultos e cinco para filhotes, foi menor do que a de outras categorias testadas, pois existem poucas marcas disponíveis no mercado.

\section{CONCLUSÕES}

Foram demonstradas diferenças importantes na composição nutricional dos alimentos econômicos, standard e super-premium, constatando-se o não cumprimento dos rótulos em parte dos alimentos avaliados e inadequações na composição nutricional de produtos.

\section{REFERÊNCIAS BIBLIOGRÁFICAS}

ASSOCIATION of the Animal Feed Control Officials. Dog and cat food substantiation methods. Canada, 2003. p.124-134. 
BRASIL. Ministério da Agricultura, Pecuária e Abastecimento. Instrução Normativa $\mathrm{n}^{\circ} 8$, de 11 de outubro de 2002. Diário Oficial [da] República Federativa do Brasil, Brasília, 2002. seção 2, p.1-6.

BURROWS, C.F.; KRONFELD, D.S.; BANTA, C.A. et al. Effects of fiber on digestibility and transit time in dogs. J. Nutr., v.112, p.1726-1732, 1982.

CARCIOFI, A.C. Proposta de normas e padrões nutricionais para a alimentação de cães e gatos. In: SIMPÓSIO SOBRE NUTRIÇÃO DE ANIMAIS DE ESTIMAÇÃO, 3., 2003, Campinas. Anais... Campinas, 2003. p.71-84.

CASE, L.P.; CAREY, D.P.; HIRAKAWA, D.A. Nutrição canina e felina: manual para profissionais. Madrid:Harcourt Brace, 1998. 424p.

COWELL, C.S.; STOUT, N.P.; BRINKMANN, M.F. et al. Making commercial pet foods. In: HAND, M.S.; THATCHER, C.D.; REMILLARD, R.L. et al. (Eds.). Small animal clinical nutrition. 4.ed. Kansas: Mark Morris Institute, 2000. p.127-146.

CROWELL, W.A.; FINCO, D.R. Frequency of pyelitis, pyelonophritis, renal perivasculitis and renal infarction in dogs. Am. J. Vet. Res., v.36, p.111-114, 1975.

CUNNIFF, P. Official methods of analysis of AOAC International. 16.ed. Gaithersburg: AOAC, 1997. v.1, p.1-45.

DEBRAEKEEKER, J.; GROSS, K.L.; ZICKER, S.C. Feeding older dogs. Geriatrics and renal diseases: nutrition management of older pets. Kansas: Hills Pet Nutrition, 1999, p.21-32.

FAHEY Jr., C.G.; MERCHEN, N.R.; CORBIN, J.E. Dietary fiber for dogs: effects of beet pup and oat fiber additions to dog diets on nutrient intake, digestibility, metabolizable energy, and digesta mean retention time. $J$. Anim. Sci., v.70, p.1169- 1174, 1992.

FINCO, D.R.; BROWN, S.A.; CROWELL, W. et al. A. Effects of dietary phosphorus and protein in dogs with chronic renal failure. Am. J. Vet. Res., v.53, p.2264-2271, 1992.

GROSS, K.L.; WEDEKIND, K.J.; COWELL, C.S. et al. Nutrients. In: HAND, M.S.; THATCHER, C.D.; REMILLARD, R.L. et al. (Eds.). Small animal clinical nutrition. 4.ed. Kansas: Mark Morris Institute, 2000. p.21-110.

HAZEWINKEL, H.A.W.; VAN DEN BROM, W.E.; KLOOSTER, A.T. et al. Calcium metabolism in great Dane dogs fed diets with various calcium and phosphorus levels. J. Nutr., v.121, p.S99-S106, 1991.

HUBER, T.L.; WILSON, R.C.; Mc GARITY, S.A Variations in digestibility of dry dog foods with identical label guaranteed analysis. J. Am. Anim. Hosp. Assoc., v.22, p.571-575, 1986 .

KIENZLE, E.; DOBENECKER, B.; EBER, S. Effect of cellulose on the digestibility of high starch versus high fat diets in dogs. J. Anim. Physiol. Anim. Nutr., v.85, p.174$185,2001$.

KIENZLE, E.; OPITZ, B.; EARLE, K.E. et al. An improved method for the estimation of energy in pet foods. J. Nutr., v.128, p.2806S-2808S, 1998.

KRONFELD, D.S. Dietary management of renal senescence and failure in dogs. Aust. Vet. J., v.71, p.328331, 1994.

LAUTEN, S.D.; COX, N.R.; BRAWNER, W.R. et al. Influence of dietary calcium and phosphorus content in a fixed ratio on grouth and development in Great Danes. Am. J. Vet. Res., v.63, p.1036-1047, 2002.

NUTRIENT requirements of cats. Washington: NRC, 1986. 78p.

PINTO, M.D. Estudo restrospectivo de afecções em cães jovens causadas por desordem metabólica ou nutricional, diagnosticada radiograficamente no período de janeiro de 1991 a dezembro de 2000. 2002. 85f. Dissertação (Mestrado em cirurgia veterinária) - Faculdade de Ciências Agrárias e Veterinárias, Universidade Estadual Paulista, Jaboticabal, SP.

PRIOR, J. Situação atual e perspectivas do mercado nacional de alimentos pet. In: SIMPÓSIO SOBRE NUTRIÇÃO DE ANIMAIS DE ESTIMAÇÃO, 3. 2003, Campinas. Anais..., Campinas: Colégio Brasileiro de Nutrição Animal, 2003. p.1-4.

ROSTAGNO, H.S.; ALBINO, L.F.T.; DONZELE, J.L. et al. Tabelas brasileiras para aves e suínos: Composição dos alimentos e exigências nutricionais. Viçosa, MG: UFV, 2000. 141p.

SCHOENMAKERS, I.; HAZEWINKEL, H.A.W.; VOORHOUT, G. et al. Effect of diets with different calcium and phosporus contents on the skeletal development and blood chemistry of growing Great Danes. Vet. Rec., v.147, p.652-660, 2000.

SCHOENMAKERS, I.; HAZEWINKEL, H.AW.; VAN DEN BROM, W.E. Excessive $\mathrm{Ca}$ and $\mathrm{P}$ intake during early maturation in dogs alters $\mathrm{Ca}$ and $\mathrm{P}$ balance without long-term effects after dietary normalization. J. Nutr., v.129, p.1068-1074, 1999.

SCOTT, D.W.; MILLER, W.H.; GRIFFIN, C.G. Doenças cutâneas nutricionais. In: _ Dermatologia de pequenos animais. Rio de Janeiro: Interlivros, 1996. p.832-842.

TRYFONIDOU, M.A.; HOLL, M.S.; VASTENBURG, $M$. et al. Hormonal regulation of calcium homeostasis in two breeds of dogs during growth at different rates. $J$. Anim. Sci., v.81, p.1568-1580, 2003. 